\title{
Four-dimensional water detection in mars using spline algorithm
}

\begin{abstract}
This finds out about has verified a brand new novelty for four-dimensional carter floor reconstruction of HRSC information. In Fact, Carter is an excellent index of water existing. The sequence of elastic transforms for B-spline is used with regard to the automatic detection algorithm of landmarks. The result suggests that 4-D axis is seen definitely as floating devices at the core of the crater. Then find out about additionally suggests that the deep of the crater is included through the potential of ice that is a magnificent evidence of liquid water lifestyles at the Mars. In other words, The examine about suggesting that 4-D axis is seen certainly as floating objects in the centre of the crater. The study about additionally suggests that the deep of the crater is blanketed through ice, which is an extraordinary proof of liquid water exists on the Mars. In conclusion, alternate of 4-D B-spline through involving elastic seriously change and landmarks algorithms ought to be a pinnacle notch promise for 4-D visualization. It can be said that the exchange of 4-D B-spline through the way of regarding elastic redecorate and landmarks algorithms may want to be a pinnacle notch promise for 4-D visualization.
\end{abstract}

Keywords: water on mars, crater, high-resolution stereo camera (hrsc), fourdimensional, b-spline.
Volume 2 Issue 5 - 2018

\author{
Maged Marghany \\ School of Humanities, Geography Section, Universiti Sains \\ Malaysia, Faculty Geospatial and Real Estate, \\ Geomatika University College, Malaysia,
}
Correspondence: School of Humanities, Geography Section, Universiti Sains Malaysia, Faculty Geospatial and Real Estate,Geomatika University College, Malaysia, Email magidupm@hotmail.com

Received: October 03, 2018 | Published: October II, 2018

\section{Introduction}

Majority water on Mars these days exists as ice, although it also exists in small quantities as vapour in the atmosphere, and sometimes as low-volume liquid brines in shallow Martian soil. The only area where water ice is seen at the surface is at the north polar ice cap. Abundant water ice additionally exists beneath the permanent carbon dioxide ice cap at the Martian South Pole and in the shallow subsurface at greater temperate conditions. More than 5 million cubic kilometres of ice have been recognized at or near the surface of cutting-edge Mars, enough to cover the whole planet to a depth of 35 meters $(115 \mathrm{ft})$.Even more, ice is probably to be locked away in the deep subsurface.

Remote sensing utilized sciences have tried to find out the Mars surface. Spectroscopic lengthy way flung sensing observations, for instance, can furnish broadly investigative compositional and mineralogical data on the Mars surfaces. Basically, three training of spectroscopic observations can be attained:

(i) Thermal emission spectra;

(ii) Reflectance spectra,

(iii) X-ray and gamma-ray spectra.

Consequently, orbital considered and thermal-IR imaging and spectroscopic analyses with the resource of the use of the Mariner 9 and Viking missions showing lavish supplementary geologically multifaceted flooring which has been formed by using the capability of the way of volcanic, and tectonics. Credibly most discovery of dendritic valley network buildings which had been originated by using the capacity of the motion of liquid water on the flooring. ${ }^{1}$ Further, thermal-IR graphics and near-IR imaging spectroscopic data, conveying evidence on the floor thermos-physical properties and mineralogy for the insufficient vicinity of the Martian floor. ${ }^{2,3}$
Consistent with Christensen, ${ }^{4}$ Kahle et al. ${ }^{5}$ the thermal emission spectra have been disbursed, for instance, to attain a evidence on disparities in continental basaltic lava flows and to confine rock abundance of the Martian surface and the thermal inertia. Moreover, Shelley ${ }^{6}$ referred to that the Hubble house Telescope (HST) seems the NASA's extraordinary dwelling observatories similarly to the Spitzer neighbourhood Telescope, the Chandra X-ray and the Compton Gamma Ray space Observatories. Those vicinity observatories are designed to work with immoderate spectra electrical energy insurance, which ranged from $20 \mathrm{keV}$ to $30 \mathrm{GeV}^{7}$ Moreover, X-ray and gamma-ray are expert to precisely extricate any object from its father or mother galaxy, and governs the physical depth in the nucleus, fingers and halo of spiral galaxies. Nonetheless, the shiny objects that extract from the spiral galaxy for an event with the beneficial aid of $\mathrm{X}$-rays and gamma rays can be tiny and range of timescales of few minutes or a few seconds. ${ }^{8-11}$

Consequently, the initial geologic studies of Mars derived from floor-primarily based telescopic observations whilst the Mars and the Earth were neighbouring and the spatial resolution arose as much as a hundred $\mathrm{km}$ decrease than the best earthbound atmospheric environment. ${ }^{2,12}$ Similarly, spacecraft orbiting Mars have brought images of canyons and flood valleys elements which endorse the life of water flows on the Mars floor. Nowadays, however, Mars is a cold, dry, wilderness-like international through a cracked ecosystem. Accordingly, no appeared organism must live on the purple Planet in the absence of water flows. ${ }^{13-16}$ The innovation of this investigation is to layout algorithm for conjuring up of crater aspects in fourdimensional by the use of High-resolution Stereo Camera (HRSC) images. In fact, HRSC can capture an image of the object in 3-D. In this view, the major hypotheses are that 4-D of any object in the region is coded into 3-D as 3D is coded into 2-D. In other words, $\mathrm{n}+1-\mathrm{D}$ is coded into n-D. Through a precise algorithm in laptop vision, the 4-D spline algorithm is believed to use in n-D simulation. Consequently, 
the find out about the interests at by means of a 4-D spline to produce 4-D of crater surfaces from the stereo of craters, which are attained through High-resolution Stereo Camera (HRSC).

\section{Four-dimensional algorithm}

In physics and mathematics, the dimension of a mathematical space is confidentially delineated, for instance, the minimal range of coordinates required to become aware of any point contained by means of it. Space and time, in classical mechanics, are various training and denote the supreme space and time. That conception of the objects is a four-dimensional (4-D) space, then again now not the one that was once originated integral to express electromagnetism. 4-D of spacetime involve activities which are not sincerely termed spatially and temporally. ${ }^{15-19}$ Otherwise, exceptionally are recognized as simulated by the motion of an observer. With these regards, any object can be power in the space of four and even higher dimensions. The key venture is to modify rationality tactics to renovate such highdimensional stuff. Following Marghany ${ }^{20}$ the $4-\mathrm{D}$ spline is used to simulate 4-D of the crater from sequences of HRSC images. Assume

$$
\tilde{\mathbf{N}}(t)=\mathbf{N}_{1 \times \mathrm{n}} \cdot \mathbf{P}_{n \times 3}=\left(\begin{array}{lll}
N_{0, \mathrm{p}}(t) & N_{0, \mathrm{p}}(t) \quad \ldots \quad \mathrm{N}_{\mathrm{n}-1, \mathrm{p}}(t)
\end{array}\right)\left(\begin{array}{cccc}
P_{0, x} & P_{0, y} & P_{0, z} & P_{0, t} \\
P_{1, x} & P_{1, y} & P_{1, z} & P_{1, t} \\
. . & . . & . . & . . \\
P_{n-1, x} & P_{n-1, y} & P_{n-1, z} & P_{n-1, t}
\end{array}\right),
$$

where $N_{i, p}(t) \quad-$ B-spline basis competencies and a manage factor matrix is $P_{n \times 3}$. To enforce the 4-D spline, the 3-D HRSC data that is assimilated at specific knot time and knot planes turn out to be temporal features. Then the detected facets in 3D HRSC can be expressed in four-D B-spline model as ${ }^{16}$

$$
R(u, v, w, t)=\sum_{i=1}^{I} \sum_{j=1}^{J} \sum_{k=1}^{K} \sum_{l=1}^{L} P_{i j k} O_{i}(u) O_{j}(v) O_{k}(w) O_{l}(t)
$$

where $O_{i}(u), O_{j}(v), O_{k}(w)$, and $O_{l}(t)$ are B-spline basis functions which blend control points $\boldsymbol{P}_{n \times 3}$ and $(I \times J \times K \times L)$ is the total number of model control points. By changing the order of B-spline summation, a more efficient approach to computing multi-dimensional B-spline model results. The simulated data included 300 frames. The fitting algorithm converged in about 30 iterations. Therefore, the total fitting process approximately took 588 seconds for 6 frames of data. An important byproduct of our approach is that at the conclusion of fitting knot solid

$u, v$, and $w$ are restrained to the interval of $u, v, w \in[0,1]$ and $S(u$, $v, w, t) \in(x, y, z) .^{21}$ This ability that the object is divided into hyper patches. The hyper patches are represented using surfaces and curves. Then, the flooring described with the aid of inserting one of $u$, $v$, and $w$ to a steady integer value which is yelled a knot plane, which is the defining the surfaces of hyper patches. ${ }^{22}$ The factors in the in the interior and on the boundary of the parametric solid is assumed with the aid of Mortenson ${ }^{22}$

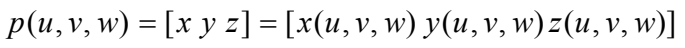

Then, the constraints of equation 1 are given by

$$
\begin{aligned}
& u_{\text {min }} \leq u \leq u_{\text {max }} \\
& v_{\text {min }} \leq v \leq v_{\text {max }} \\
& w_{\text {min }} \leq w \leq w_{\text {max }}
\end{aligned}
$$

The 4-D B-spline curve is well-defined by means of the subsequent equation $\left(n_{k}=n\right)$ :

to frames of data, a 4-D model $\mathrm{S}(u, v, w$, and $t)$ is determined. Given two solids $\mathrm{S}\left(u, v, w, t_{0}\right)$ and $\mathrm{S}\left(u, v, w, t_{1}\right)$, a 3 -D B-spline interpolated motion field is immediately gen erated by employing the computation in. ${ }^{21,23}$

$$
V(u, v, w)=S_{1}\left(u, v, w, t_{1}\right)-S_{0}\left(u, v, w, t_{0}\right)
$$

The 4-D shape mannequin is self-regulating of the intrinsic description of 3-Dand 4-D figures when landmarks are existing. It can be effortlessly prolonged to the 4-D case after normalizing the variety of phases per exclusive sub-images of all frames in a subjective range of data. In this learn about we used 30 frames, and using the landmark advent algorithm continually for each section of 30 frames over time t. Then, a collection of elastic transforms are implemented to bring the transmitted landmarks on the pinnacle of the crater surface. The following equation is used for a series of elastic transforms as

$$
\mathrm{T}_{\text {elastic }}(u, v, w, t)=[u, v, w, t]^{T}+\sum_{i=1}^{I} \sum_{j=1}^{J} \sum_{k=1}^{K} \sum_{l=1}^{L} P_{i j k} O_{i}(u) O_{j}(v) O_{k}(w) O_{l}(t) M_{i+l, j+m, k+n, L+o}
$$

where $M$ denotes $N_{u} \times N_{v} \times N_{w} \times N_{t}$ mesh for control point of $[u, v, w, t]^{T}$ which are identified within a volume of the dimension of $[u, v, w, t]$. The elastic transforms were achieved with a series of B-spline changes by four levels of meshes, $(4 \times 4 \times 4),(6 \times 6 \times 6),(8 \times$ $8 \times 8)$, and $(16 \times 16 \times 16)$. Its allied control points for each mesh level, $\mathrm{M}$ are adjusted to minimize the space between the distorted landmarks and the number of the object surface.

\section{Data sets}

Consistent with Marghany ${ }^{15}$ ESA's space probe Mars explicit was launched on June 2, 2003, through a Soyuz-Fregat rocket from Baikonur Cosmodrome in Kazakhstan. It entered the elliptical orbit of Mars on December 25, 2003. The orbit of Mars-specific has the most distance of $10,530 \mathrm{~km}$ above the Martian floor and $330 \mathrm{~km}$ on adjoining approach (Figure 1). This physical geometry promises for examinations of the Martian moons Phobos and Deimos along with measuring its atmospheric profile. Consequent Marghany ${ }^{19,20}$ the crater information is assimilated from HRSC. The HRSC is recording the Mars in 3-D two with a resolution of about 10 meters (Figure 2). In preliminary degree, the mission of HRSC is aimed nearer to searching out liquid waters and existence interior the Red Planet's surface. Thus far, about $75 \%$ of the Martian surface has been blanketed in 3-D. Precise areas emerge as imaged at $2 \mathrm{~m}$ resolution. That is due to the digital sensor encompasses extremely-excessive-decision telephoto lens barring excellent choice Channel (SRC), which is imaging objects to a few meters in measurement (DLR 2015). 


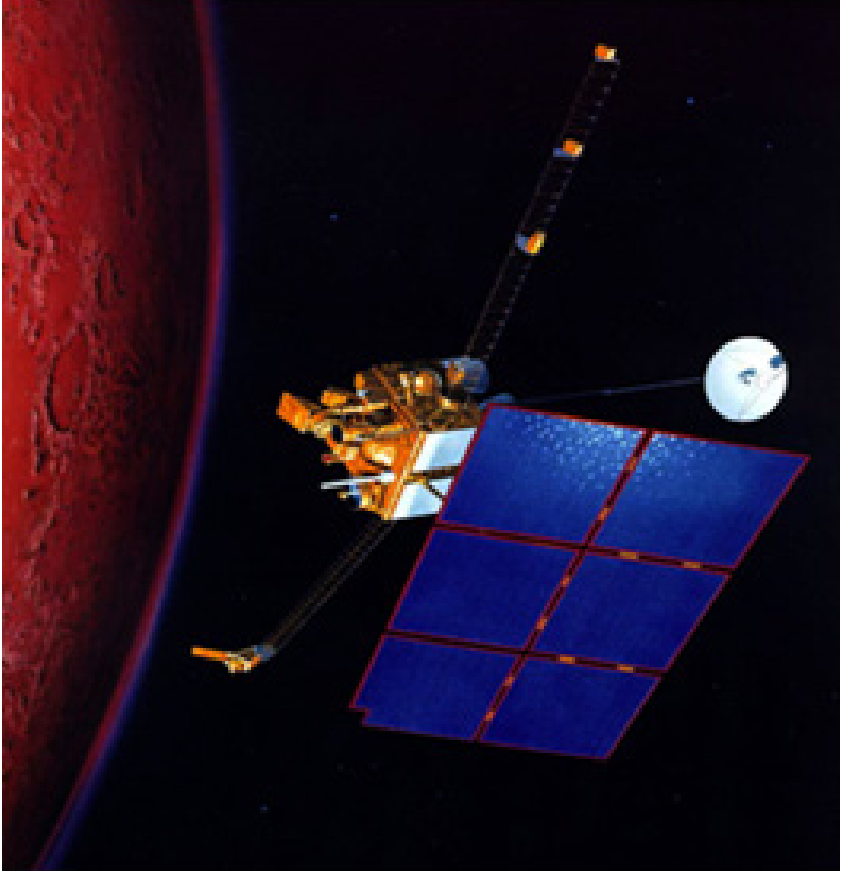

Figure I Mars Express in orbit around Mars.

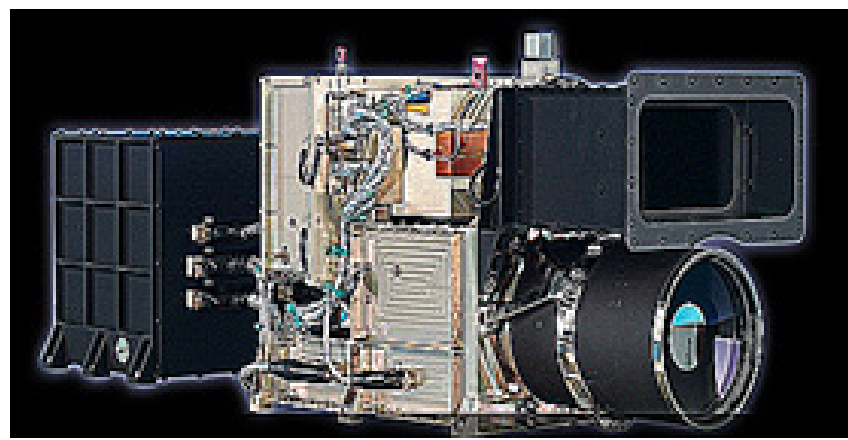

Figure 2 High-resolution Stereo Camera HRSC.

According to DLR (2015), three $\mathrm{km}$ wide in the centre of the photo strips; the surface information is imaged with a decision of $2.3 \mathrm{~m}$ in step with the pixel. The SRC recordings offer a geological background of the region, that's delivered by the high-decision imageries that are received by the stereo head. ${ }^{20}$ Consequently, Figure 3 displays the HRSC camera scanning system. Further, the SRC head is composed of a reflecting telephoto lens and a CCD array sensor. For instance, each sensor information the identical item on the surface at a one of a sort perspective. Three-dimensional images are generated with the aid of five of the image strips. The last 4 of the 9 line sensors are geared up with extraordinary colouration filters for recording multi-spectral facts. ${ }^{15}$ Consistent with Marghany \& Mansor $^{15}$ the HRSC camera system, which simplest weighs 20 kilograms, has two digital camera heads. The excessive resolution Stereo head, which consists of $9 \mathrm{CCD}$ line sensors hooked up in parallel to the lower back of a lens. Approximately the shortest distance of 270 $\mathrm{km}$ from the spacecraft. Subsequently, the determination of the nine photo strips at this height is $12 \mathrm{~m}$ for each of the 5184 seven-micron rectangular pixels. The sensor has a swath of $52 \mathrm{~km}$ and the minimal strip length of $300 \mathrm{~km}$. The latter depends on the spacecraft's facts garage and transmission capability. The terrific resolution Channel (SRC) is used as a magnifying glass.

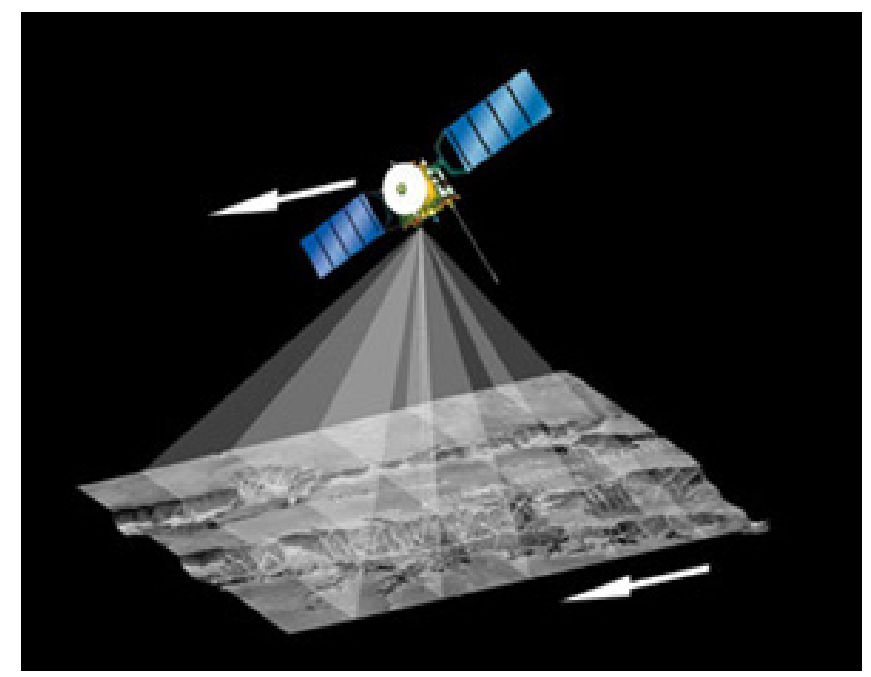

Figure 3 HRSC camera scanning system.

\section{Results and discussion}

The dominant visible features which exist in Mars surface are the Carters. Crater may be created through two cosmic bodies crashed each different, as an instance, a meteorite putting a planet. It can be moreover long-established through the volcanic profession. The one of a kind form of carter is subsidence crater, which is occurring from an underground because of a nuclear explosion. A crater-like pattern fashioned via erosion, which is diagnosed as Machetes. Furthermore, a comfort crater triggered via a phreatic outburst or explosion is described as a crater. Therefore, Mars is a terrestrial planet which incorporates minerals. These minerals contain silicon and oxygen, metals, and exclusive factors which naturally structure rock. The Mars floor is ordinarily produced from tholeiitic basalt. With those regards, the Mars is needed elegant strategies to understand its complex nature and developments formation. In fact, man has an incredible hobby to find out Mars to be an alternative agreement of the Earth. Presently, Marghany ${ }^{19,20}$ have pragmatic the fourth-dimensional set of rules to examine the geological carter factors of the Mars.

Evidently, the craters DEM inside the Hellas Basin are exposed in Figure 4. It is thrilling to find that the DEM varies between $-3 \mathrm{~km}$ to $6 \mathrm{~km}$. Craters geographical location is $29^{\circ} \mathrm{S}$, and $68^{\circ} \mathrm{E}$ in the northern rim of Hellas basin. This archive data acquired on 8 July 2004 by way of the HRSC digital camera. Corresponding to Marghany ${ }^{19}$ the Hellas Basins have formed among between 3.8 and 4.1 billion years in the past, after a terrific asteroid hit the Mars surface. These Hellas basins have been modified by the possessions of wind motion, ice, water and volcanic hobby for the reason that they had been shaped. As well, HRSC become able to imagine the craters with the ground resolution is about $15 \mathrm{~m}^{7}$ in line with pixel with $25 \mathrm{~km}$ across. Figure 5 , indicates the simulated crater DEM which ranged from $-3 \mathrm{~km}$ to $3 \mathrm{~km}$. The bottom point of DEM of $-3 \mathrm{~km}$ is located in deep in the crater.

Consistent with Marghany ${ }^{19,20}$ European Space Agency (ESA) has established the Mars-specific mission which aimed at analyzing the surface, subsurface, environment and ionosphere of the Mars. The gadgets are expended for floor and subsurface research are implicated

(i) High-resolution Stereo Camera (HRSC);

(ii) Visible and Infrared Mineralogical Mapping Spectrometer 
(OMEGA) and; Subsurface Sounding Radar Altimeter (MARSIS). Consequently, the atmosphere and Ionosphere gadgets are

(iii) Energetic Neutral Atoms Analyzer (ASPERA);

(iv) Planetary Fourier Spectrometer (PFS) and

(v) Ultraviolet and Infrared Atmospheric Spectrometer (SPICAM). Moreover, the radio hyperlink instrument which is Mars Radio Science Experiment (Mars).

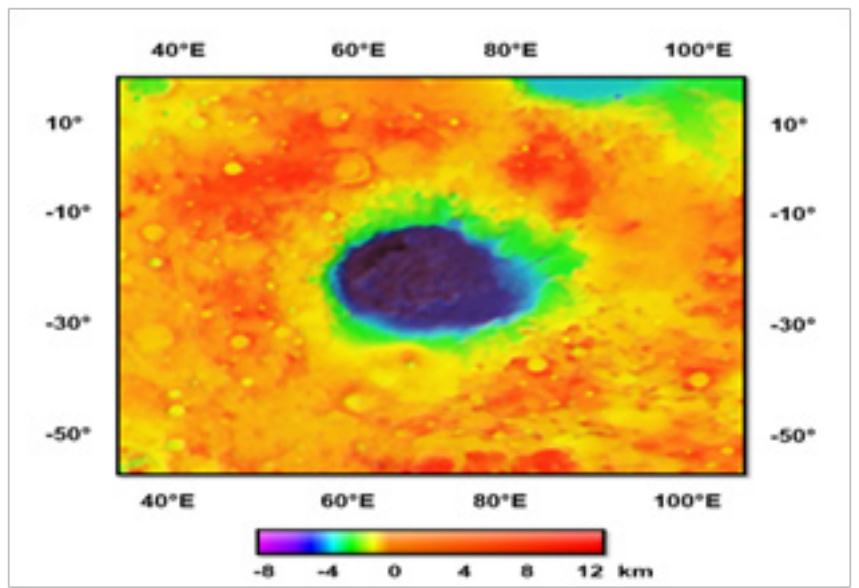

Figure 4 Craters DEM Hellas Basin.

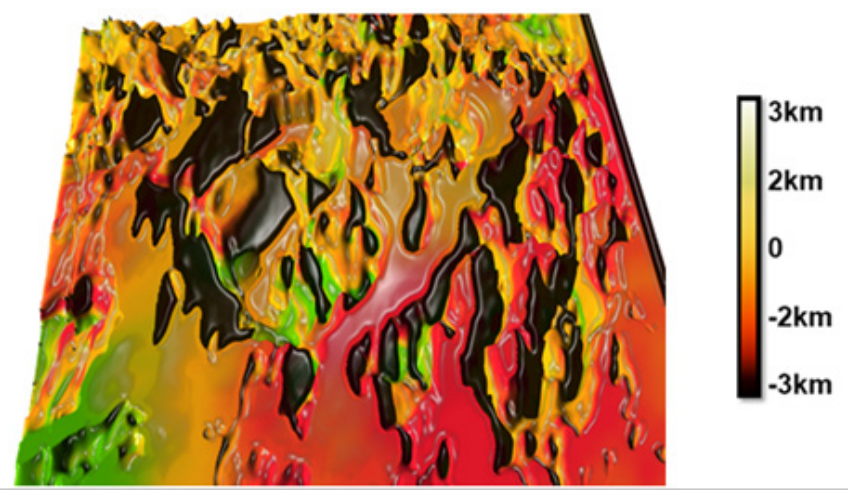

Figure 5 3-D of crater simulated by B-spline.

Conclusively, HRSC grants 3-D images of geological facets of the Mars, which consists of the surface, crust, and interior of the Mars. Certainly, the time period planetary geology science is to find out about the stable components of moons and planets. The bulk of the two craters are about $25 \mathrm{~km}$ across. Therefore, the morphology of many facets in the Hellas Basin and its environment strongly suggests the presence of ice and glaciers. The HRSC can produce 3-D of Mars geological facets due to the fact of the High-Resolution Stereo head works on push-broom mode: sensors, photo a line on the planet floor perpendicular to the ground track of the spacecraft and matter on the orbital movement of the spacecraft to reposition them as they greatest a sequence of photos recognized as an image swath. This agrees with the studies of Hartmann, ${ }^{3}$ Carr ${ }^{1}$ and Marghany. ${ }^{19,20}$ Figure 6 suggests the 4-D of the crater which simulated using a 4-D spline. It is fascinating to locate that 4-D layer is considered truly as floating in the centre of the crater. 4-D is engendered routinely through the aid of innovations. Indeed, innovations are routinely created and transmitted to 4-D information with the aid of skill of rigid alignment, distance-based merging, and B-spline transforms. The energy of the series of elastic transforms which are recognized inside an extent of a 4-D dimension. This is simply obvious in Figure 6. Additionally, the sequence of B-spline changes are carried out through the elastic transforms to gather precisely $4-\mathrm{D}$ of crater volumetric. The landmarks are compulsory to be constantly scattered on the crater surface and concentrated ample consequently that their triangular mesh is adjoining to the original crater surface. Predominantly, landmarks positioned on crater surfaces have to fit up to every pixel. This learn about confirming the work completed by way of Waks et al. ${ }^{24}$ Duda, ${ }^{25}$ Marghany ${ }^{26}$ and Marghany ${ }^{20}$ Marghany \& Mansor. ${ }^{15}$

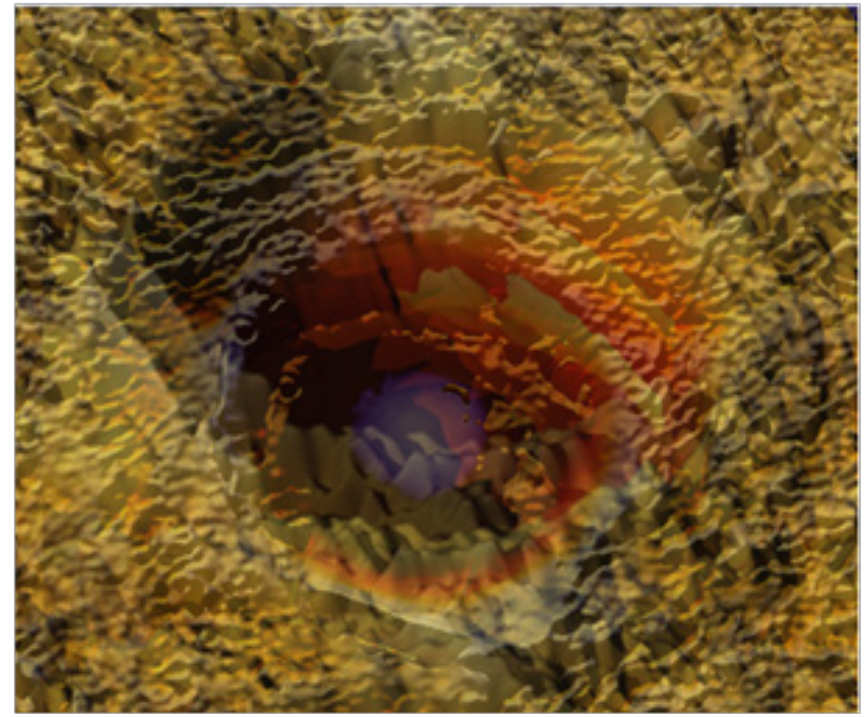

Figure 6 4-D crater simulated by B-spline.

Moreover, the deep of the crater is dominated by ice, which is the proof of liquid water existence. This should attribute to that inside $-3 \mathrm{~km}$ in the deep of the crater; the atmospheric stress is about $89 \%$ greater than the surrounding of the crater. This discovering consents with Marghany ${ }^{19}$ and ESA. ${ }^{27}$ Truthfully, the 4-D spline algorithm is separated 3-D craters from HRSC data into hyper patches. Subsequently, the hyper patches are carried out the use of surfaces and curves. Formerly, the floor labelled by way of tracing one of 3-D elements to a consistent integer price which is the major of hyper patches. ${ }^{28,29}$

\section{Conclusion}

A carter is a most visual characteristic which exists on Mars surface. The crater can be created through the potential of two cosmic our bodies crashed each other, for instance, a meteorite putting a planet. The foremost contribution of this work is to simulate 4-D of Mars carters. In doing so, 3-D information of HRSC used to be acquired and then the 4-D spline algorithm carried out to sequences of HRSC archives with a magnificent frame of times. The find out about suggests that B-spline can determine the 4-D go with the waft sample which ought to indicate for the existence of water. The sequence of elastic transforms for B-spline is used with involving computerized detection algorithm of landmarks. The examine about suggesting that 4-D axis is seen certainly as floating objects in the centre of the crater. The study about additionally suggests that the deep of the crater is blanketed through ice, which is an extraordinary proof of liquid water exists on the Mars. In conclusion, alternate of 4-D B-spline through involving elastic seriously change and landmarks algorithms ought to be a pinnacle notch promise for 4-D visualization. 


\section{Acknowledgemnets}

None.

\section{Conflict of interest}

The authors declare that there is no conflict of interest.

\section{References}

1. Carr M. The surface of Mars. Cambridge, UK: Cambridge University Press; 2006.

2. Rencz AN. Remote sensing for the earth sciences: manual of remote sensing Volume 3. John Wiley and sons; 1999.

3. Hartmann W. A Traveler's Guide to Mars: The Mysterious Landscapes of the Red Planet. New York: Workman Publishing; 2003.

4. Christensen PR. The spatial distribution of rocks on Mars. Icarus. 1986;68(2):217-238.

5. Kahle AB, Palluconi FD, Christensen PR. Thermal emission spectroscopy: Application to the Earth and Mars. Remote Geochemical Analysis: Elemental and Mineralogical Composition. Cambridge Univ Press. 1993;99-120.

6. Shelley C. NASA's Great Observatories. NASA; 2008.

7. Marghany M. Four-dimensional mars crater reconstructions using 4-D spline algorithm. $38^{\text {th }}$ Asian Conference on Remote Sensing - Space Applications: Touching Human Lives, ACRS. 2017.

8. Evans LG, Reedy RC, Trombka JI. Introduction to planetary remote sensing gamma ray spectroscopy. In: Pieters CM, et al. Editors. Remote Geochemical Analysis: Elemental and Mineralogic Composition. Cambridge Univ Press. 1993:167-198.

9. Yin LI, Trombka JI, Adler, et al. X-ray remote sensing techniques for geochemical analysis of planetary surfaces. In: Pieters CM, et al. Editors. Remote Geochemical Analysis: Elemental and Mineralogical Composition. Cambridge Univ Press. 1993:199-212.

10. Burns RG. Origin of Electronic Spectra of Minerals in the Visible-Near Infrared Region. In: Pieters CM, et al. Editors. Remote Geochemical Analysis: Elemental and Mineralogical Composition. Cambridge: Cambridge Univ Press. 1993:1-29.

11. Gaffey SJ, McFadden LA, Nash DB. Ultraviolet, visible, and nearinfrared reflectance spectroscopy: Laboratory spectra of geologic materials. In Remote Geochemical Analysis: Elemental and Mineralogical Composition. 1993:43-71.

12. Martin LJ, James PB, Dollfus A, et al. Telescopic observations: Visual, photographic, polarimetric. Univ of Ariz Press, Tucson. 1992:34-70.

13. De Vaucouleurs G. Physics of the Planet Mars. London: Faber and Faber; 1954.
14. Mutch TA, Arvidson RE, Head JW, et al. The Geology of Mars. Princeton Univ Press Princeton NJ; 1976.

15. Marghany M. Mansor S. Four-dimensional B-spline Algorithm for MARs Crater Reconstructions. CD of $37^{\text {th }}$ Asian Conference on Remote Sensing (ACRS). Colombo, Sri Lanka: Galadari Hotel; 2016:1-6.

16. Amini AA, Chen Y, Curwen RW, et al. Coupled B-Snake Grids and Constrained Thin-Plate Splines for Analysis of 2-D Tissue Deformations from Tagged MRI. IEEE Transactions on Medical Imaging. 1998;17(3):344-356.

17. Marghany M. Polarised AIRSAR along track interferometry for shoreline change modeling. Geoscience and Remote Sensing Symposium, 2003 IGARSS'03. Proceedings. 2003 IEEE International. 2003;2:945-947.

18. Marghany M. Modelling shoreline rate of changes using holographic interferometry. International Journal of Physical Sciences. 2011;6(34):7694-7698.

19. Marghany M. 4-D geological feature reconstructions in Mars using High Resolution Stereo Camera (HRSC). CD of $36^{\text {th }}$ Asian Conference on Remote Sensing (ACRS 2015), Manila, Philippines. 2015.

20. Marghany M. Fourth-dimensional optical hologram interferometry of rapid eye for Japan Tsunami effects. 36th Asian Conference on Remote Sensing: Fostering Resilient Growth in Asia, ACRS 2015. 2015;1-6.

21. Amini AA, Radeva P, Li D. Measurement of 3D motion of myocardial material points from explicit B-surface reconstruction of tagged MRI data. Medical Image Computing and Computer-Assisted Intervention. 1998;110-118

22. Mortenson ME. Geometric Modeling. John Wiely \& Sons, NewYork. $1985 ; 1-36$

23. Marghany M. Advanced Remote Sensing Technology for Tsunam Modelling and Forecasting. New York: CRC Press, Taylor \&Francis Group; 2018.

24. Waks E, Prince J, Douglas. Cardiac Motion Simulator for Tagged MRI Mathematical Methods in Biomedical Image Analysis. 1996;182-191.

25. Duda RO, Hart PE, Stork DG. Pattern Classification. John Wiley \& Sons Inc; 2001 .

26. Marghany M. Hologram interferometric SAR and optical data for fourth-dimensional urban slum reconstruction. Proceedings of $35^{\text {th }}$ Asian conference on remote sensing, Nay Pyi Taw. 2014.

27. ESA. Craters within the Hellas Basin. Space in images. 2015.

28. Radeva P, Amini A, Huang J. Deformable B-solids and implicit snakes for 3D Localization and tracking of SPAMM MRI Data. Computer Vision and Image Understanding. 1997;66(2):163-178.

29. Sheehan W. Planets and Perception. Univ Arizona Press; 1988. 